\title{
Metamodeling and optimization of the THF process with pulsating pressure
}

Marco Bucconi, and Matteo Strano

Citation: AIP Conference Proceedings 1960, 110002 (2018); doi: 10.1063/1.5034959

View online: https://doi.org/10.1063/1.5034959

View Table of Contents: http://aip.scitation.org/toc/apc/1960/1

Published by the American Institute of Physics

\section{Articles you may be interested in}

Investigation of the effects of process and geometrical parameters on formability in tube hydroforming using a modular hydroforming tool

AIP Conference Proceedings 1960, 150002 (2018); 10.1063/1.5035009 


\title{
Metamodeling and Optimization of the THF Process with Pulsating Pressure
}

\author{
Marco Bucconi ${ }^{1, a)}$ and Matteo Strano ${ }^{1, b)}$ \\ ${ }^{1}$ Dipartimento di Meccanica, Politecnico di Milano, Via La Masa 1, Milan, Italy \\ a) Corresponding author: marco.bucconi@polimi.it, Tel: +39-0223998534 \\ b) matteo.strano@polimi.it
}

\begin{abstract}
Tube hydroforming is a process used in various applications to form the tube in a desired complex shape, by combining the use of internal pressure, which provides the required stress to yield the material, and axial feeding, which helps the material to flow towards the bulging zone. In many studies it has been demonstrated how wrinkling and bursting defects can be severely reduced by means of a pulsating pressure, and how the so-called hammering hydroforming enhances the formability of the material. The definition of the optimum pressure and axial feeding profiles represent a daunting challenge in the designing phase of the hydroforming operation of a new part. The quality of the formed part is highly dependent on the amplitude and the peak value of the pulsating pressure, along with the axial stroke. In this paper, a research is reported, conducted by means of explicit finite element simulations of a hammering THF operation and metamodeling techniques aimed at optimizing the process parameters for the production of a complex part. The improved formability is explored for different factors and an optimization strategy is used to determine the most convenient pressure and axial feed profile curves for the hammering THF process of the examined part. It is shown how the pulsating pressure allows the minimization of the energy input in the process, still respecting final quality requirements.
\end{abstract}

\section{HAMMERING TUBE HYDROFORMING INTRODUCTION}

The conventional tube hydroforming (THF) process has been studied several times with the aim of optimizing the loading curves, i.e. the pressure vs. time and axial feed vs. time curves [1]. In fact, the outcome of the THF process is strongly history-dependent and FEM simulation is widely used in order to reduce the time and cost of the process planning phase. The design and optimization of loading curves is required in order to avoid the typical THF defects: a) excessive thinning and bursting, when pressure increases with no sufficient axial feeding; b) wrinkling, when the axial feeding is too large with respect to the internal pressure. The typical pressure vs. time loading curve for any THF process is a monotonously non-decreasing profile; pressure must obviously increase because of the tube strain hardening and shape changing. Unfortunately, as pressure increases, not only the tube wall thickness decreases in the expansion zones, but the axial feeding, which is crucial to the process success for many typical THF parts becomes more and more difficult, because of an increasing level of frictional resistance. The idea of tube hydroforming with an oscillation of internal pressure has been proposed by some authors and tested by in previous works [2]. They observed an improvement of formability. The main reason for the improvement of formability is thought to be the reduction of friction at the die-tube interface, especially in the guiding zone. This reduction facilitates axial feeding with reduced risk of wrinkling. However, some authors proved that, in FEM simulation, pulsating pressure improves the formability, even for frictionless tube hydroforming [3]. THF with pulsating pressure is also called "hammering" THF. Unfortunately, if process design, i.e. the selection of process parameters of conventional THF, is a delicate task, which requires expert users and FEM based optimization, the introduction of the pulsating parameters makes the optimization problems more complex. Few works have studied the hammering THF with the purpose of optimizing the process parameters. In a recent paper [4], the authors have shown, with a Taguchi-based sensitivity analysis, that the number of pressure peaks significantly influences the success of the THF operation of a T-shape, while the amplitude of the pressure oscillation is not as important as expected. However, the number of oscillations cannot be 
easily increased, in the industrial practice, because of technological constraints of the pressure intensifying machines. In the present paper, with respect to an industrial case study, the hammering THF process will be optimized with a metamodel based optimization algorithm. Four process parameters will form the vector $\underline{x}$ of design variables to be optimized. This vector includes the amplitude of the pressure oscillation, while the frequency of the oscillations (i.e. the number of oscillations peaks) is selected to a constant value, due to technological considerations.

\section{CASE STUDY DEFINITION}

The aim of this study is to develop a methodology to optimize the process parameters of a tube hydroforming with pulsating pressure process in order to minimize the energy input, under certain conditions of final quality of the part. In this study, the hydroforming process is simulated using a commercial finite element software, PAM-STAMP. A metamodel is constructed based on the results of 162 simulations where four different parameters are randomly changed within a given range. The goal of the optimization will be to minimize the energy spent for the process, under technological and feasibility constraints.

\section{Tube Hydroformed Part}

The hydroformed part analyzed in this paper is represented by component used in the paper making industry, consisting of a tube with a bulged end. Its dimensions are given in Fig. 1.

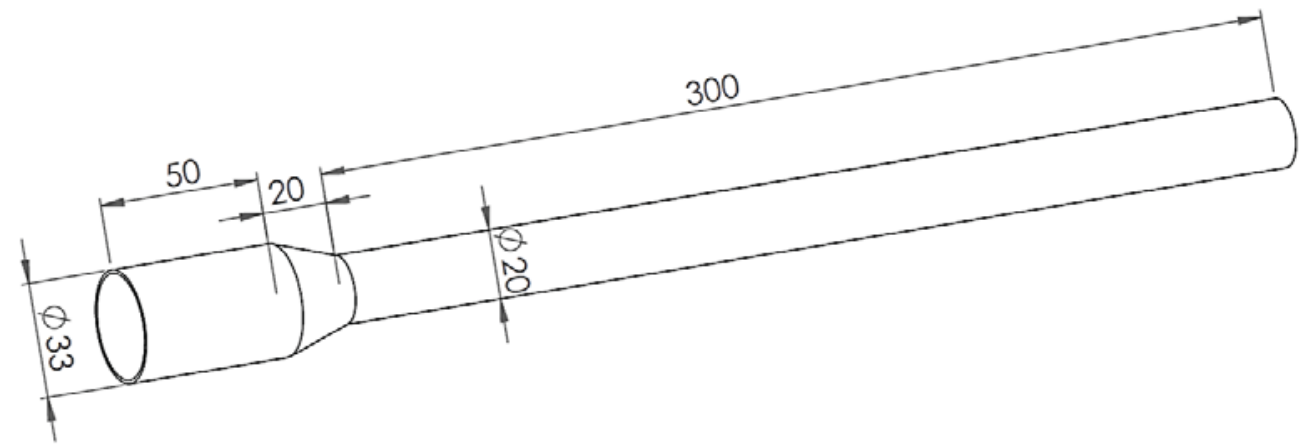

FIGURE 1. Final Hydroformed part

Since the part is small enough, it is possible to form two components simultaneously and trim them at a later stage, improving the productivity of the process. In simulation, in order to save computational time, but still be coherent with the actual production of two simultaneous parts, a plane of symmetry was placed at the undeformed tube end (the right end side in Fig. 1). The blank is represented by a AISI 316L tube of 20mm in diameter and $2 \mathrm{~mm}$ in thickness. The material proprierties are represented in Table 1. The hardening curve was modeled using the Krupkowski law.

TABLE 1. AISI 316L proprieties

\begin{tabular}{cccccc}
\hline $\mathrm{E}[\mathrm{GPa}]$ & $v$ & $\rho\left[\mathrm{Kg} / \mathrm{mm}^{3}\right]$ & $\varepsilon_{0}$ & $\mathrm{~K}[\mathrm{GPa}]$ & $\mathrm{n}$ \\
\hline 210 & 0.3 & 7.8 & 0.001 & 1.07 & 0.203 \\
\hline
\end{tabular}

The tool set of the tube hydroforming process consist of: upper die, lower die and two axial punches. The punches move axialy, enhancing the flow of the material in the bulged zone of the tube. The velocity curve of the punch is defined linerly by four points. The integral of the velocity profile over time represets the displacement of the punch $\Delta \mathrm{X}(\mathrm{mm})$. Both velocity and displacement profiles are shown in Fig. 2. 


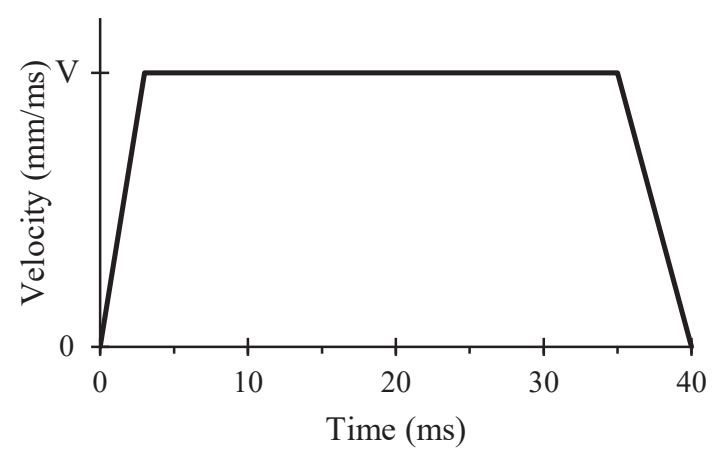

(a)

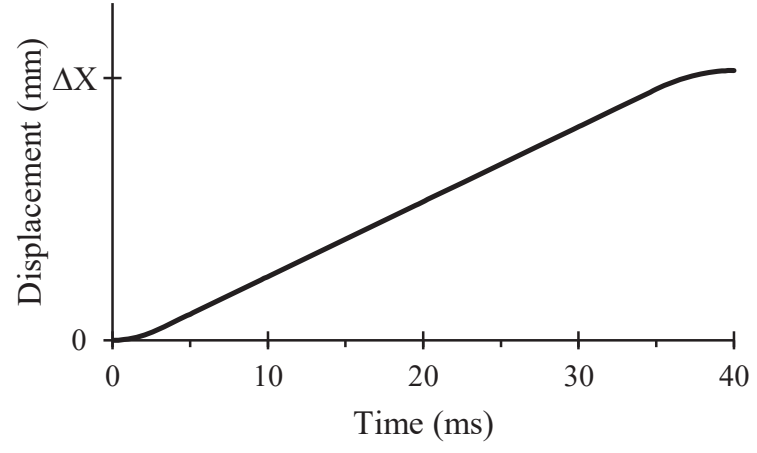

(b)

FIGURE 2. (a) Profile of the velocity of the axial punch vs time; (b) profile of the displacement of the axial punch vs. time

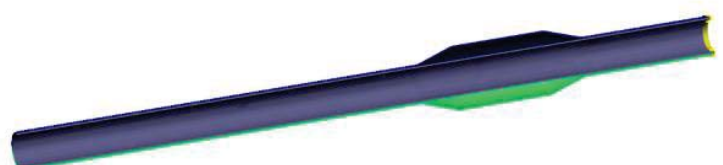

(a)

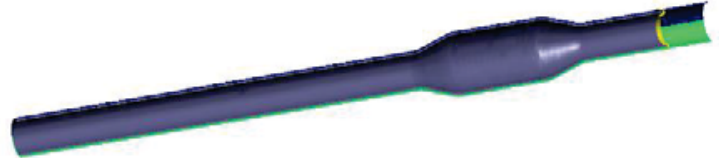

(b)

FIGURE 3. (a) Example of the first state of the forming simulation; a symmetry plane has been placed at the left tube end; (b) example of final state of the forming simulation

Figure 3 shows the first and final state of a simulation of the hydroformed part. It can be noticed that some excess material near the the axial punch is needed to form the bulge on the part; this material needs to be trimmed away after the hydroforming process. To simulate the hydroforming action a time dependent pressure is applied at the tube inner wall. The pressure curve, Fig. 4 , is defined by four points: $0 \mathrm{MPa}$ at $0 \mathrm{~ms}$, Bulging pressure (fixed at $95 \mathrm{MPa}$ ) $\mathrm{BP}$ at $3 \mathrm{~ms}$, Forming pressure FP at $35 \mathrm{~ms}$ and Calibration pressure $\mathrm{CP}$ at $40 \mathrm{~ms}$. The actual process time duration is 40 seconds, but the simulated time is accelerated by a factor 1000 . Furthermore, if hammering hydroforming is used, the pressure curve is dependent on the pressure amplitude $\triangle \mathrm{P}(\mathrm{MPa})$, which pulsates in the time interval between 3 and $35 \mathrm{~ms}$ with a frequency $f=0.25 \mathrm{kHz}$ (corresponding to $0.25 \mathrm{~Hz}$ for the actual process):

$$
p(t)=B P-\frac{\Delta P}{2}+\frac{(F P-B P) \cdot(t-3)}{35-3}+\frac{\Delta P}{2} \cdot \cos (2 \cdot \pi \cdot f \cdot(t-3))
$$

The contact between the tube and the dies is supposed to be highly lubricated and therefore a coefficient of friction of 0.05 was assigned to the dies.

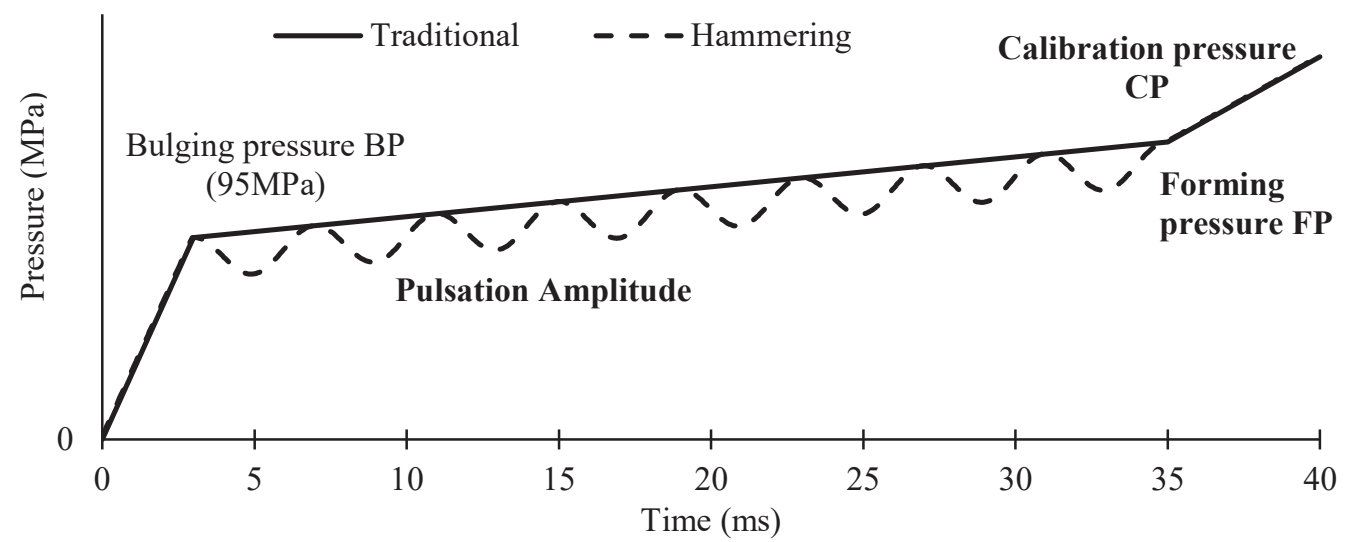

FIGURE 4. Example of the pressure vs. time profile 


\section{Simulation Plan}

A total of 162 different simulations were carried out, randomly changing the four design parameters: $\triangle \mathrm{P}, \mathrm{FP}, \mathrm{CP}$ and $\Delta \mathrm{X}$. The given technological limits for the parameters were defined as:

- $\quad$ Forming pressure (FP): 95-200 MPa

- Calibration pressure (CP): 95-200 MPa

- Pulsating pressure amplitude $(\Delta \mathrm{P}): 0-20 \mathrm{MPa}$

- Punch displacement $(\Delta \mathrm{X}): 0-70 \mathrm{~mm}$

For every simulation, the presence of cracks was detected and the distance tube-die $\delta$ at the end of the process, the thinning $t$ and external energy expenditure $E$ were measured. Only 124 of the simulations proved, from the Forming Limit Diagram, to be feasible without cracks, and therefore suitable to be embedded in the metamodel. In Fig. 5, all the combinations of the different parameters used are displayed.

For each simulation, a number equal to 0 was assigned in case of unfeasibility of the process, in terms of presence of cracks, excessive thinning and excessive maximum distance between the formed part and the die. On the contrary, a value of 1 was assigned in case of complete feasibility. Fitting these data with a binary logistic regression model helps to identify some zones of the design variables where the process is feasible. In Fig. 6, the areas highlighted with a darker color represent a combination of two design variables where the process is more likely to be feasible.
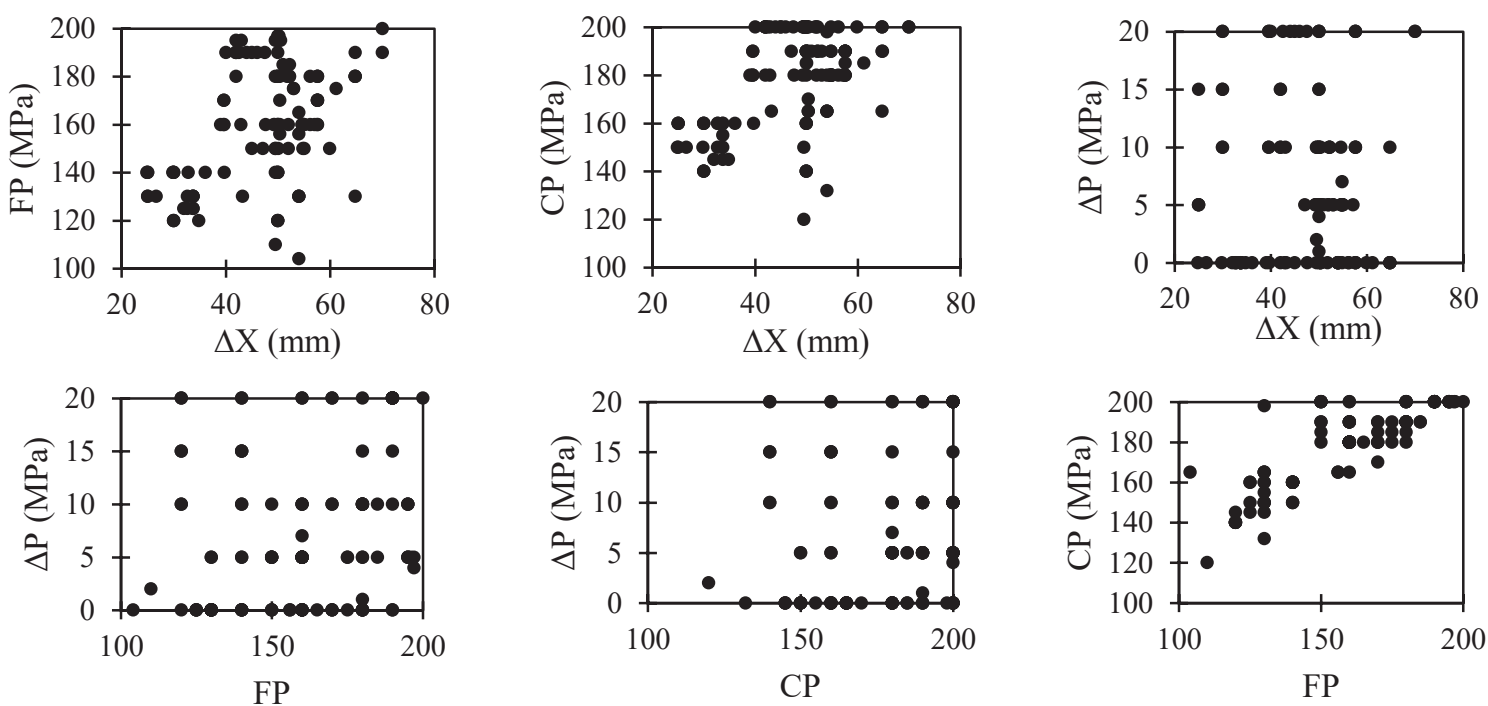

FIGURE 5. Combination of the different parameters used in the simulation plan

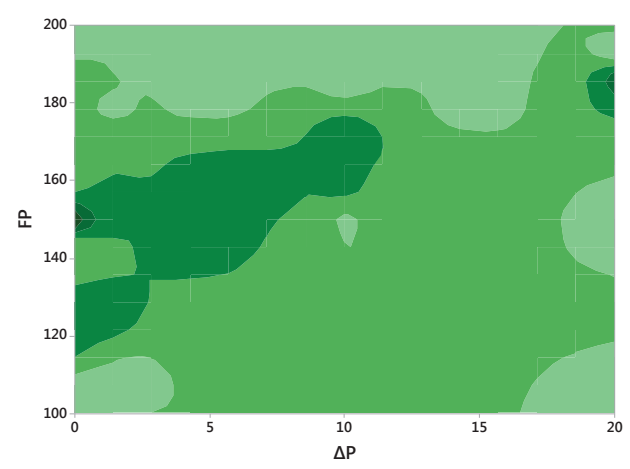

(a)
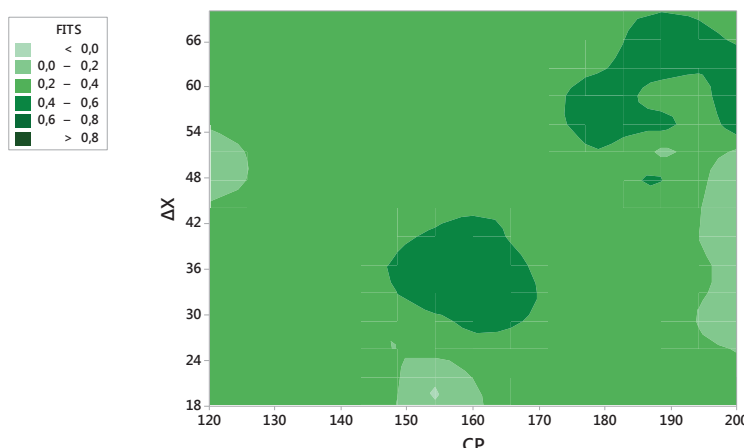

(b)

FIGURE 6. Contour plot of feasibility of the process: (a) FP vs. $\Delta \mathrm{P}$; (b) $\Delta \mathrm{X}$ vs. CP 


\section{METAMODELLING AND OPTIMIZATION}

A kriging metamodel has been built using the vector $\underline{\mathrm{x}}$ of the 4 design variables (where $\mathrm{x}_{1}=\Delta \mathrm{P}, x_{2}=\mathrm{FP}, \mathrm{x}_{3}=C P$, $\left.\mathrm{x}_{4}=\Delta \mathrm{X}\right)$ and the vector $\mathrm{y}$ of 3 response variables, where $\mathrm{y}_{1}=\delta, \mathrm{y}_{2}=t, \mathrm{y}_{3}=E$. In the Kriging metamodel, the predicted response is the realization of a regression model $F(\underline{x})$ and a correlation function $z(\underline{x})$.

$$
\underline{\hat{y}}=F(\underline{x})+z(\underline{x})=\beta_{0}+\sum_{i=1}^{n} \beta_{i} \cdot f_{i}(\underline{x})+z(\underline{x})
$$

where $\beta_{i}$ are the $n$ linear regression coefficients, $f_{i}(\underline{x})$ are $n$ regression terms, $z(\underline{x})$ is an exponential correlation function (stochastic process) with zero mean. The $f_{i}(x)$ regression terms are polynomial functions of the design variables of maximum order 2, i.e. the $F(\underline{x})$ regression function is quadratic. The correlation function is built in order that the metamodel interpolates the results of the FEM simulations at the tested design sites. The Kriging interpolator also yields an estimation of the interpolation errors at the untested sites, which can be expressed as a vector function of expected mean square errors $\hat{\underline{\varphi}}_{y}(\underline{x})$.

An optimization algorithm has been implemented in MATLAB using the built in fmincon function, which enables to minimize a multivariable nonlinear constrained function. The constrained optimization problem can be defined as follows:

$$
\begin{aligned}
& \min _{\underline{x}} y_{3} \\
& \text { s.t. } \quad\left\{\begin{array}{c}
\underline{x}_{L B}<\underline{x}<\underline{x}_{U B} \\
y_{i}<0.3 \forall i=1,2 \\
\sum_{i=1}^{3} \underline{\hat{\varphi}_{y i}} / y_{i}<0.3
\end{array}\right.
\end{aligned}
$$

where $\underline{x}_{L B}$ and $\underline{x}_{U B}$ are the minimum and maximum allowed values of $\underline{x}$ (already defined in the previous section), as a result of the technological limits of the process. A set of constraints has been added to take into account the feasibility of the process in terms of final quality. The aim of the study is to minimize the energy input of the THF process avoiding an excessive thinning of the tube $\left(t=y_{2}<0.3\right)$ and an under-formed part, expressed by the value of the maximum distance $\delta$ between the tube wall and the die at the final state of the simulation $\left(\delta=y_{1}<0.3\right)$. Additionally, a constraint was added to take into account a limit on the mean square error of the predictor function:

The solution given by the fmincon function represents a local minimum of the predicted response function, and it is highly sensitive to the search starting point $\underline{\mathrm{x}}$. Therefore, the minimization algorithm was embedded in a loop and at each iteration a different starting point was tested. Since the design points used in the simulation plan have the lowest Mean Square Error, it is logical to assume that a local minimum found near those points could be more reliable. Hence, the first group of starting points was set to equal to the design sites tested in the simulations. In addition to those, in order to explore the entire 4D-space represented by the four design variables, a random generator was used to create 2000 different starting points within the technological boundaries.

The five best results of the algorithm in terms of energy input are saved and verified by further FEM simulations. In case of large discrepancy between the modelled results and the simulation, particularly due to an insufficient amount of initial data, it is necessary to increase the number of starting data used to construct the metamodel. A solution can be achieved through an iterative reconstruction of the metamodel done by including the results of the FEM simulation at the five minimum results coming from the minimization algorithm. Eventually, when the initial data are dense enough the coherence between the metamodel and the simulations will be guaranteed. In Fig. 7 the flowchart of the optimization process is presented.

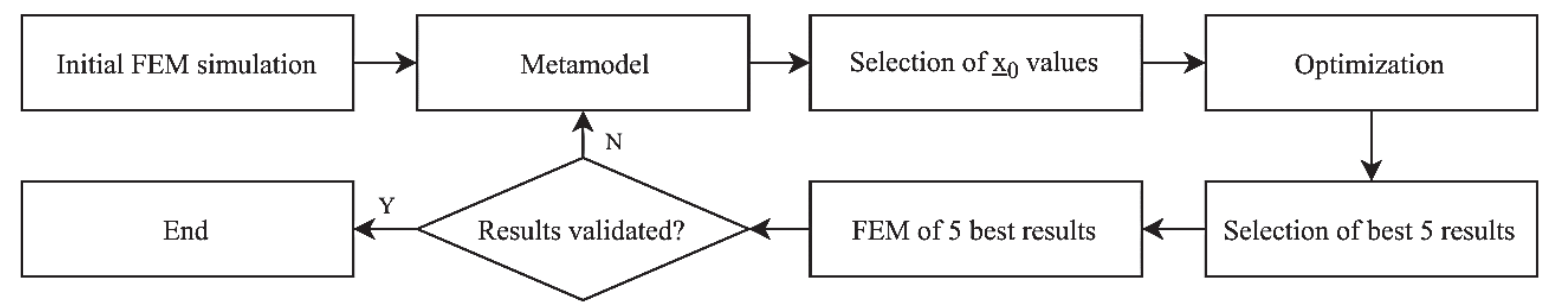

FIGURE 7. Flowchart of the optimization process 


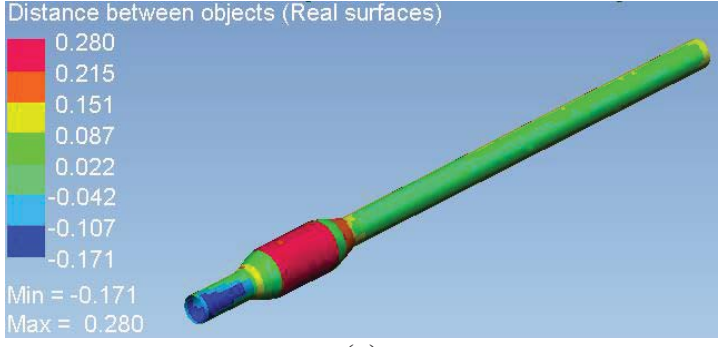

(a)

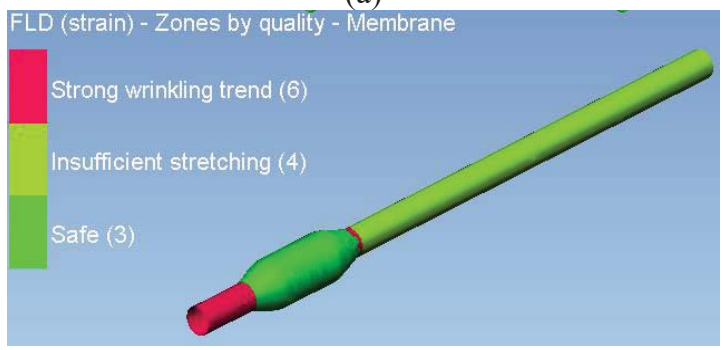

(c)

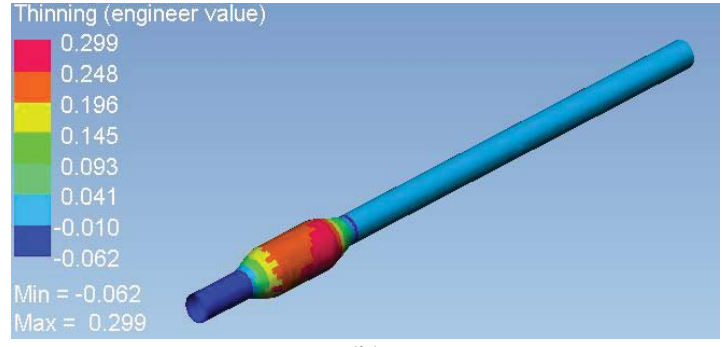

(b)

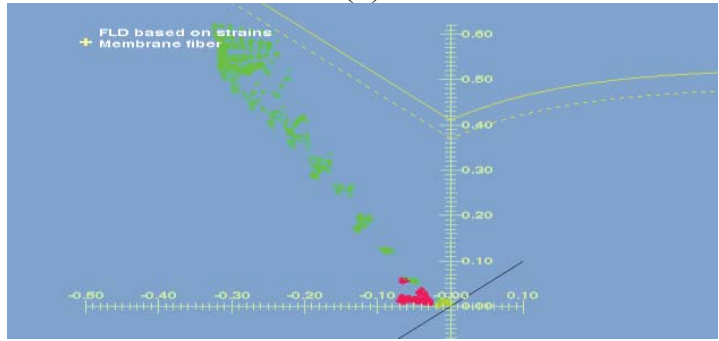

(d)

FIGURE 8. Contour plots of the optimized solution of: (a) tube-die distance; (b) Thinning of the tube wall; (c) and (d) FLD

Out of the five best results of the first run of the minimization algorithm: one presented a crack and the remaining four differed from the corresponding validation FEM simulation in the range of $2 \%$ and $42 \%$. Therefore, these results were embedded in metamodel and the minimization algorithm launched again. These adjustments of the metamodel were sufficient to obtain at a second run consistent results. In fact, the five best results of the optimization algorithm of the new metamodel differed from their FEM validation between $1 \%$ and $4 \%$. Out of the five results, the one that assured the respect of the quality constraints also in the FEM validation stage (Fig. 8) represents the end of the optimization search. The minimum value of energy input, $3.20 \cdot 10^{6} \mathrm{~J}$, is obtained with the following design variables:

- $\quad$ Forming pressure (FP): $140 \mathrm{MPa}$

- $\quad$ Calibration pressure (CP): $160 \mathrm{MPa}$

- $\quad$ Pulsating pressure amplitude $(\Delta \mathrm{P}): 10 \mathrm{MPa}$

- $\quad$ Punch displacement $(\Delta \mathrm{X}): 29.84 \mathrm{~mm}$

\section{CONCLUSIONS}

In this work, an optimization strategy aimed at reducing the total energy input for a tube hammering hydroforming process has been developed and applied to an industrial case study. The influence of four design variables has been explored at a FEM simulation stage. The three main results can be summed up as follows.

- The optimization algorithm provided a solution of design variables that minimizes the energy input and respected the quality constraints.

- The minimization algorithm provided reliable results, later validated by means of FEM simulations.

- The improved formability observed with pulsating pressure allowed the reduction of energy input in the THF process, though respecting quality constraints on the thinning, final shape and absence of cracks.

\section{REFERENCES}

1. M. Strano, S. Jirathearanat, S. G. Shr, T. Altan, J. Mater. Process. Technol. 146, 2004, pp. 130-136.

2. K. Mori, T. Maeno, S. Maki, Int. J. Mach. Tools Manuf. 47, 2007, pp. 978-984.

3. M. Loh-Mousavi, M. Bakhshi-Jooybari, K. I. Mori, K. Hyashi, Proc. Inst. Mech. Eng. Part B J. Eng. Manuf. 222 , 2008, pp. 1139-1146.

4. A. Ashrafi, K. Khalili, Proc. Inst. Mech. Eng. Part B J. Eng. Manuf. 230, 2016, pp. 1203-1212. 